\title{
IL-8 Enhances Therapeutic Effects of BMSCs on Bone Regeneration via CXCR2- Mediated PI3k/Akt Signaling Pathway
}

\author{
Aijun Yang ${ }^{a, b, c}$ Yanzhu Lu $u^{a, b, c}$ Junchao Xing ${ }^{a, b, c}$ Zhilin Li $i^{a, b, c, e}$ \\ Xiaolong Yin ${ }^{a, b, c}$ Ce Dou ${ }^{a, b, c}$ Shiwu Dong ${ }^{a, b, c, d} \quad$ Fei Luo ${ }^{a, b, c}$ \\ Zhao Xie ${ }^{a, b, c}$ Tianyong Hou ${ }^{a, b, c}$ Jianzhong $\mathrm{Xu}^{\mathrm{a}, \mathrm{b}, \mathrm{c}}$
}

aDepartment of Orthopedics, National \& Regional United Engineering Laboratory of Tissue Engineering, Southwest Hospital, the Third Military Medical University, Chongqing, ${ }^{b} \mathrm{Center}$ of Regenerative and Reconstructive Engineering Technology in Chongqing City, Chongqing, 'Tissue Engineering Laboratory of Chongqing City, Chongqing, dDepartment of Biomedical Materials Science,

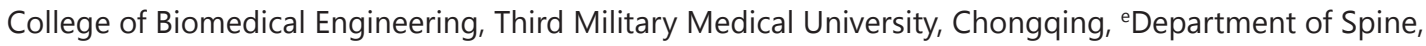
Lanzhou General Hospital, Lanzhou Command of CPLA, Lanzhou, China

\section{Key Words}

Interleukin-8 (IL-8) • Tissue engineered bone (TEB) • Bone marrow mesenchymal stem cells $(\mathrm{BMSCS}) \cdot \mathrm{CXCR2} \cdot \mathrm{PI} 3 \mathrm{k} \cdot \mathrm{Akt}$

\begin{abstract}
Background/Aims: Tissue engineering bone transplantation with bone marrow mesenchymal stem cells (BMSCs) is an effective technology to treat massive bone loss, while molecular regulation of the bone regeneration processes remains poorly understood. Here, we aimed to assess the role of interleukin-8 (IL-8) in the recruitment of host cells by seeded BMSCs and in the bone regeneration. Methods: A transwell assay was performed to examine the role of IL-8/CXCR1/CXCR2/PI3k/Akt on the migration potential of hBMSCs. The in vitro chondrogenic differentiation of hBMSCs was assessed by examination of 2 chondrogenic markers, Sox 9 and type 2 collagen (COL2). mBMSCs were used in tissue engineered bone (TEB) with/without IL- 8 implanted into bone defect area with CXCR2 or Akt inhibitors. Density and Masson staining of the regenerated bone were assessed. The chondrogenesis was assessed by expression levels of associated proteins, Sox9 and COL2, by RT-qPCR and by immunohistochemistry. Results: IL-8 may trigger in vitro migration of hBMSCs via CXCR2-mediated PI3k/Akt signaling pathway. IL-8 enhances osteogenesis in the TEB-implanted bone defect in mice. IL-8 induces chondrogenic differentiation of hBMSCs via CXCR2-mediated PI3k/Akt signaling pathway in vitro and in vivo. Conclusions: IL-8 enhances therapeutic effects of MSCs on bone regeneration via CXCR2mediated PI3k/Akt signaling pathway.

Aijun Yang, Yanzhu Lu and Junchao Xing contributed equally to this work.

Jianzhong Xu and

Tianyong Hou

Dep. of Orthopedics, National \& Regional United Engineering Lab. of Tissue Engineering, Southwest Hospital, the Third Military Medical University; 29 Gaotanyan Street, Shapingba District, Chongqing 400038, (China)

Tel. +8613883502972, E-Mail prof_jianzhongxu@163.com / tianyonghou@yeah.net
\end{abstract}

KARGER 


\section{Cellular Physiology Cell Physiol Biochem 2018;48:361-370 \begin{tabular}{l|l} 
DOI: 10.1159/000491742 & O 2018 The Author(s). Published by S. Karger AG, Basel \\
www.karger.com/cpb
\end{tabular}}

Yang et al.: a Role of IL-8 in BMSCs-Mediated Bone Regeneration

\section{Introduction}

Complex fractures can be caused by traffic accidents, resection of bone tumors, and debridement of a wide range of bone infections [1-5]. When the bone loss exceeds a volume threshold, the bone fails to repair the defect through autologous regeneration, and requires therapeutic approaches to maintain the limb length and restore limb function. Currently, the treatment of massive bone loss includes autologous bone transplantation, allograft bone transplantation, tissue engineering bone transplantation, et al. Although autologous bone graft is an ideal method of repair, the limited supply of bone source as well as bone surgery-associated infection, pain and other complications prevented its wide application [6]. Tissue engineered bone provides new strategies for the treatment of bone defects $[7,8]$. However, the current tissue engineering bone suffers from several shortcomings like lack of osteoconductive and limited sources of seed cells [9]. The seed cells used for engineering bone are mainly osteoblasts, human umbilical cord mesenchymal stem cells, embryonic stem cells, adipose-derived stem cells, transgenic stem cells and bone marrow mesenchymal stem cells (BMSCs). BMSCs are easy to be obtained in a non-invasive way. The strong proliferative capacity in vitro and multiple differentiation potential of BMSCs make them ideal seed cells for massive bone repair. BMSCs that are used for decalcified bone matrix scaffold tissue engineering bone have been shown to have osteoinductivity and osteogenic, while its osteogenic potential remains poorly understood $[10,11]$.

The role of seed cells in the process of osteogenesis has been thought to stem from the proliferation and differentiation into osteoblasts by the seed cells on the tissue engineering bone to complete the bone repair [12-15]. However, recent studies showed that the seed cells contributed less to the later repaired tissues and most cells that participate into bone repair were derived from the host cells that had migrated to the site of injury to promote wound healing $[13,16-18]$. It was suggested that seed cells release a variety of cytokines such as IL-2, IL-6, IL-8, SDF-1, and TNF to promote the migration and differentiation of host cells $[13,16-18]$. Under physiological conditions, the concentration of IL-8 is extremely low, but could be rapidly induced by proinflammatory cytokines such as TNFa and IL-1b [19]. Thus, the role of IL-8 in the recruitment of host cells in the bone repair is interesting to be investigated.

IL-8 is a member from CXC cytokine family [20]. IL-8 can be produced by a variety of cells, such as macrophages, epithelial cells, airway smooth muscle cells and endothelial cells [20]. MSCs also secrete IL-8, which regulates MSC function in both autocrine and paracrine manners [20]. IL-8 effects through binding to its receptors CXCR1 or CXCR2 [20]. Both CXCR1 and CXCR2 are members of the G-protein coupled receptor (GPCR) family and have a similar transmembrane membrane structure [20]. Activation of CXCR1 or CXCR2 by IL-8 activates the intracellular PI3K and MAPK pathway, and then exert effects in a cell-type-dependent manner [21]. PI3K pathway activation leads to Akt phosphorylation, which is critical for cell survival, angiogenesis and cell migration [22-24]. However, the role of IL-8 in osteogenesis repair through its receptor CXCR1 or CXCR2 remains to be elucidated [24-29]. A previous study has shown that IL-8 can recruit BMSCs to repair cartilage defects [30]. Another study showed that IL-8 promotes BMSCs to differentiate into cartilage via CXCR2 [31]. Therefore, in the current study, we aimed to study the effects of IL-8 on therapeutic effects of MSCs on bone regeneration and its underlying mechanisms.

\section{Materials and Methods}

Human BMSCs and in vitro migration experiments

Bone marrow was obtained from healthy volunteers who signed the informed consent form. Fresh bone marrow was diluted with 0.1 M phosphate buffer (Gibco, Carlsbad, CA, USA) and the nucleated cells in bone marrow were obtained by density gradient centrifugation. The obtained cells were cultured in DMEM/ F12 medium (GE Healthcare Life Sciences HyClone Laboratories, Utah, UT, USA) containing 12\% fetal bovine 


\section{Cellular Physiology Cell Physiol Biochem 2018;48:361-370 \begin{tabular}{l|l} 
DOI: 10.1159/000491742 & $\begin{array}{l}\text { O } 2018 \text { The Author(s). Published by S. Karger AG, Basel } \\
\text { www.karger.com/cpb }\end{array}$
\end{tabular}}

Yang et al.: a Role of IL-8 in BMSCs-Mediated Bone Regeneration

serum in an incubator at a concentration of $5 \%$ carbon dioxide at $37^{\circ} \mathrm{C}$ to obtain adherent spindle-shaped swirl-like growth cells, which could be induced to differentiate into Bone, fat and cartilage. Adherent culture was used to obtain bone marrow mesenchymal stem cells (hBMSCs). Transwell chambers ( $8 \mu \mathrm{m}$ pore size, Corning Co., NY, USA) were added to $200 \mu \mathrm{l}$ of hBMSCs at a concentration of $1 \mathrm{X} 10^{5} / \mathrm{ml}$ and then $500 \mu \mathrm{l}$ of drug-containing DMEM/F12 medium was added to a 24-well plate. Control, IL-8, anti-CXCR1 antibody (aCXCR1, R\&D System, Los Angeles, CA, USA), SB225002 (Selleck Chemicals, Houston, TX, USA), LY294002 (Selleck Chemicals) and MK22-6 (Selleck Chemicals), weere added in 3 replicate wells. After 36 hours, cells in the upper part of the small cell membrane were wiped off and fixed with paraformaldehyde. The cells were then stained with DAPI (Sigma-Aldrich, St. Louis, MO, USA) and examined.

\section{Cartilage-induced culture of hBMSCs}

The third generation hBMSCs were used to differentiate into cartilage. The chondrogenic-inducing medium was a medium containing $1 \times$ insulin iron selenide (Gibco), $1 \%$ double antibody, $50 \mathrm{mg} / \mathrm{mL}$ ascorbic acid and $10 \mathrm{ng} / \mathrm{mL}$ transforming growth factor-beta3. In order to form stereotactic culture of hyaline cartilage, $0.5 \mathrm{ml}$ cell suspension with a concentration of $5 \times 10^{5}$ cells / $\mathrm{ml}$ was transferred to a 15 $\mathrm{ml}$ polypropylene centrifuge tube and centrifuged at $150 \mathrm{~g}$ for $5 \mathrm{~min}$ at room temperature. MK-2206 was used as 400uM, SB225002 was used as $10 \mathrm{uM}$, which were added to IL-8 $(100 \mathrm{ng} / \mathrm{ml})$. The incubation was done in a $5 \% \mathrm{CO}_{2}$ incubator at $37{ }^{\circ} \mathrm{C}$. Twenty-four hours after the cell mass aggregation, the bottom of the centrifuge tube was flicked so that the cartilage ball from the bottom of the tube is suspended in the liquid. Chondrocytes were collected for analysis of cartilage-associated transcription factors and marker proteins after 7 days' culture. Pathological analysis of cartilage ball collected at 28 days.

\section{Preparation of mBMSCs}

Four-week-old male fvb mice (Third Military Medical University Animal Experimental Center) were killed, after which tibial bone marrow cells were obtained under aseptic conditions. Mouse bone marrow mesenchymal stem cells (mBMSCs) were obtained by whole-bone culture, as described [32]. The phenotype of mBMSCs were evaluated by flow cytometry analysis of the surface markers Sca-1, CD90, CD105, CD34, CD45 and HLA-DR, and inducible differentiation of mBMSCs into osteocytes, adipocytes and chondrocytes with Osteocyte Differentiation Tool (American Type Culture Collection (ATCC), Rockville, MD, USA; Catalog number: PCS-500-052), Adipocyte Differentiation Toolkit (ATCC; Catalog number: PCS-500-050) and Chondrocyte Differentiation Tool (ATCC; Catalog number: PCS-500-051), respectively. Alcian blue staining, Von kossa staining and Oil red $\mathrm{O}$ staining were performed for detecting differentiated chondrocytes, osteocytes and adipocytes, respectively.

\section{Construction of mouse tissue engineered bone (TEB) with/without mouse IL-8}

The mBMSCs were transduced with adeno-associated virus (AAV) carrying recombinant mouse IL-8 to obtain mBMSCs/IL-8 under the control of a CMV promoter. Briefly, the control scrambled construct or mouse IL-8 coding sequence was cloned into a pAAV-CMV-RFP plasmid (Clontech, Mountain View, CA, USA). To generate AAVs, HEK293T cells (ATCC) were co-transfected with $10 \mu \mathrm{g}$ of the prepared plasmids and $5 \mu \mathrm{g}$ each of packaging plasmids using Lipofectamine 3000 (Invitrogen, St. Louis, MO, USA). The viruses were purified using CsCl density centrifugation and the titrated with a quantitative densitometric dot-blot assay. For generating TEB, a $2 \mathrm{X} 2 \mathrm{X} 2 \mathrm{~mm}$-size demineralized bone matrix (DBM) was prepared. The DBM was placed in ultra-low adsorption 24-well plates (Cornin Co.) and $10 \mu \mathrm{l}$ of mBMSCs or mBMSCs/IL-8 at a concentration of $1 \mathrm{X}^{\circ} 0^{6} / \mathrm{ml}$ was applied to the DBM and placed in a $5 \% \mathrm{CO}_{2}$ incubator at $37^{\circ} \mathrm{C}$ for 1 hour. After turning the DBM upside down, $10 \mathrm{ul}$ of mBMSCs or mBMSCs/IL-8 at a concentration of $1 \mathrm{X} 10^{6} / \mathrm{ml}$ was again dropped onto the DBM and placed in a $5 \% \mathrm{CO}_{2}$ incubator at $37^{\circ} \mathrm{C}$ for 1 hour. Media is changed every 1-2 days. TEB was removed after one week of culture.

\section{Tissue engineering bone repair of long bone defects in mice}

The male eGFP mice of 12 weeks of age (Third Military Medical University Animal Experimental Center) were anesthetized by intraperitoneal injection of $0.5 \%$ sodium pentobarbital at a dose of $15 \mathrm{mg} /$ $\mathrm{kg}$. The animals were fixed in a prone position and skin preparation was performed in the conventional leg surgery area with iodophor disinfection. The right limbs knees were put at $90^{\circ}$, after which a longitudinal incision of about $1 \mathrm{~cm}$ in length was made on the lateral side of the knee joint. The skin and subcutaneous 


\section{Cellular Physiology Cell Physiol Biochem 2018;48:361-370 \begin{tabular}{c|c} 
DOI: 10.1159/000491742 & $\begin{array}{l}\text { O } 2018 \text { The Author(s). Published by S. Karger AG, Basel } \\
\text { www.karger.com/cpb }\end{array}$
\end{tabular}}

Yang et al.: a Role of IL-8 in BMSCs-Mediated Bone Regeneration

tissue were incised and the white muscle line was revealed. Blunt dissection of the rectus femoris muscle and semitendinosus muscle space along the white line was performed to fully expose the femur (Including the greater trochanter), and the screws were firmly fixed in the femoral shaft resection of $2 \mathrm{~mm}$ length of the backbone and periosteum, to allow the TEB to be implanted into the bone defects. The right lower limb was protected against immobilization to allow free movement, eating and drinking. SB225002 (1 mg / kg / 2 days) and MK-2206 (100 mg / kg / week) were given in the corresponding groups. Morphological histological analysis of the samples taken 14 days after operation.

\section{Micro-CT analysis}

Specimens were fixed in paraformaldehyde for 48 hours, after which MicroCT examination was applied.

\section{Histology and immunohistochemistry}

After the sample was fixed in paraformaldehyde for 48 hours, it was decalcified in 10\% EDTA decalcification solution for 2 weeks. After decalcification was complete, internal fixation was removed and paraffin specimens were prepared into 4um-thickness consecutive sections, for H\&E, Masson and Safranin 0 staining, which were evaluated by three pathologists independently. For immunohistochemistry, sections were washed three times with PBS and then incubated with goat anti-COL2 (Santa Cruz Biotechnology, Dallas, TX, USA), goat anti-Sox 9 (Santa Cruz Biotechnology) overnight at $4{ }^{\circ} \mathrm{C}$. After washing with PBS, antigoat secondary antibody was shown as a red fluorescent donkey. Primary antibody and secondary antibody dilution ratio was 1:100. DNA were stained by DAPI (Sigma-Aldrich). The images were obtained by Lycra laser confocal microscope and analyzed.

\section{Quantitative RT-PCR}

The quantitative RT-PCR assay is summarized as follows. Total RNA was extracted by total RNA extraction kit (Qiagen, Valencia, CA, USA) according to instructions. Total RNA is transcribed by reverse transcription kit (Qiagen). The primers used for RT-qPCR were: GAPDH (sense: AGGGCTGCTTTTAACTCTGGT, anti-sense: GGCATGGACTGTGGTCATGAG); Sox9 (sense: CACAAGAAAGACCACCCGGA, antisense: GGAAATGTGCGTCTGTTCGG); COL2 (sense: GGGCAGAGGCAGGAAACTAA, antisense: TGTAGGACACACGCAGTTCC). The average of three cycles was used to calculate gene expression, with GAPDH as an internal control.

\section{Statistical analysis}

All values represent the mean \pm standard deviation (SD). Statistical analysis of group differences was carried out using a one-way analysis of variance (ANOVA) test (SPSS 19.0, Chicago, IL, USA) followed by the Fisher's Exact Test to compare two groups. A value of $\mathrm{p}<0.05$ was considered statistically significant after Bonferroni correction.

\section{Results}

\section{IL-8 triggers in vitro migration of hBMSCs via CXCR2}

We purchased hBMSCs and put the cells in culture (Fig. 1A). In order to examine whether IL-8 (IL-8) plays a key role in the migration of hBMSCs, as well as through which receptor (CXCR1 or CXCR2) hBMSCs may respond to IL-8, we performed a transwell migration study. The hBMSCs were cultured in the upper chamber of the transwell, while the lower chamber contained either control media (CTL), or media with IL-8 (IL-8), or media with IL-8 and antibody against CXCR1 (aCXCR1; IL-8+aCXCR1), or media with IL-8 and SB225002 (a specific inhibitor for CXCR2; IL-8+ SB225002), or media with IL-8 and LY294002 (a specific inhibitor for PI3k; IL-8+ LY294002), or media with IL-8 and MK-2206 (a specific inhibitor for Akt; IL-8+ MK-2206), respectively (Fig. 1B). The migrated cells after 24 hours' culture were determined, shown by representative images (Fig. 1C), and by quantification (Fig. 1D). Our data showed that IL-8 significantly increased the migrated hBMSCs, while suppression of CXCR2 by SB25002, but not suppression fo CXCR1 by aCXCR1, abolished the effects of IL-8 (Fig. 1C-D). Moreover, suppression of PI3k/Akt signaling also abolished the effects of IL-8 


\section{Cellular Physiology and Biochemistry}

(Fig. 1C-D). Together, these data suggest that IL-8 may trigger in vitro migration of hBMSCs via CXCR2-mediated PI3k/ Akt signaling pathway.

IL-8 induces chondrogenic differentiation of hBMSCS via CXCR2-mediated PI3k/Akt signaling pathway in vitro In order to assess in vitro chondrogenic differentiation of hBMSCs by IL-8, hBMSCs were treated with either control media (CTL), or media with IL-8 (IL8), or media with IL-8 and SB225002 (IL-8+ SB225002), or media with IL-8 and MK-2206 (IL-8+ MK-2206), respectively (Fig. 2A). Then the hBMSCs were subjected to chondrogenic differentiation media in micromass culture for 7 days, followed by alcian blue staining (Fig. 2B). The chondrogenic differentiation was assessed by examination of two chondrogenic markers, Sox9 and COL2, by RT-qPCR in the differentiated hBMSCs (Fig. 2C). Our data showed that IL-8 signifi-

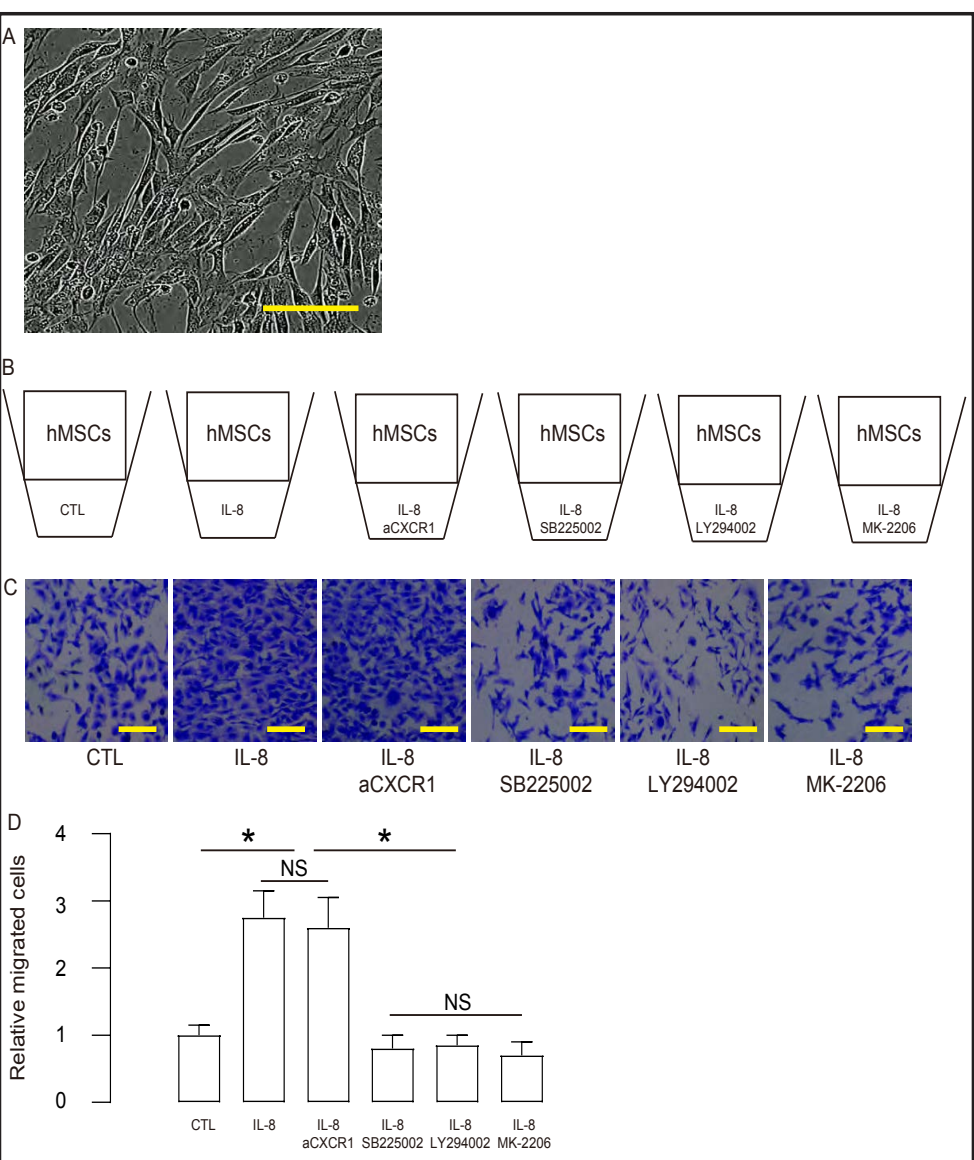

Fig. 1. IL-8 triggers in vitro migration of hBMSCs via CXCR2. (A) hBMSCs in culture. (B) A transwell migration study in which hBMSCs were cultured in the upper chamber of the transwell, while the lower chamber contained either control media (CTL), or media with IL-8 (IL-8), or media with IL-8 and antibody against CXCR1 (aCXCR1; IL-8+aCXCR1), or media with IL-8 and SB225002 (a specific inhibitor for CXCR2; IL-8+ SB225002), or media with IL-8 and LY294002 (a specific inhibitor for PI3k; IL-8+ LY294002), or media with IL-8 and MK-2206 (a specific inhibitor for Akt; IL-8+ MK2206), respectively. (C-D) The migrated cells after 24 hours' culture were determined, shown by representative images (C), and by quantification (D). ${ }^{*} p<0.05$. NS: non-significant. $N=5$. Scale bars are 20uM. cantly increased the Sox 9 and COL2 levels in differentiated hBMSCs, while suppression of CXCR2 by SB25002, or suppression of Akt signaling by MK-2206, similarly abolished the effects of IL-8 (Fig. 2C). These data suggest that IL-8 induces chondrogenic differentiation of hBMSCs via CXCR2-mediated PI3k/Akt signaling pathway in vitro.

\section{Isolation and characterization of $m B M S C S$}

Next, we isolated mouse BMSCs (mBMSCs) for in vivo study (Fig. 3A). The surface marker analysis for Sca-1, CD90, CD105, CD34, CD45 and HLA-DR confirmed an BMSC phenotype (Fig. 3B). Also, mBMSCs were able to be induced to differentiate into chondrocytes, adipocytes or osteocytes (Fig. 3C). Hence, the phenotype of the isolated mBMSCs was confirmed.

IL-8 enhances osteogenesis in the TEB-implanted bone defect in mice

Mouse tissue engineered bone (TEB) with/without overexpression of mouse IL-8 (mBMSCs; mBMSCs/IL-8) was prepared (TEB; TEB/IL-8). A 2mm-length resection of 
bone was generated at the right femur of the eGFPtransgenic mice, after which TEB, or TEB/IL-8, or TEB with SB225002, or TEB with MK-2206 was implanted into the bone defect. Morphological histological analysis of the samples were taken 14 days after operation by microCT (Fig. 4A). The density of the regenerative bone was determined, showing that IL-8 significantly increased the density of the regenerative bone, while either SB225002 or MK-2206 significantly decreased it (Fig. 4B). Masson staining was performed, showing that IL-8 significantly increased the collagen (Masson+ area) in the regenerated bone, while either SB225002 or MK-2206 significantly decreased it (Fig. 4C). Thus, IL-8 enhances osteogenesis in the injured mouse bone via CXCR2-mediated PI3k/ Akt signaling pathway.

\section{IL-8 enhances}

chondrogenesis associated proteins in the injured mouse bone Finally,

the chondrogenesis in the regenerated bone was assessed by examination of two chondrogenic markers, Sox 9 and COL2, by RT-qPCR (Fig. 5A), and by immunohistochemistry (Fig. 5B). Our data showed that IL-8 significantly increased the Sox9 and COL2 levels in the regenerated bone, while suppression of CXCR2 by SB25002, or suppression of Akt signaling by MK2206, similarly abolished

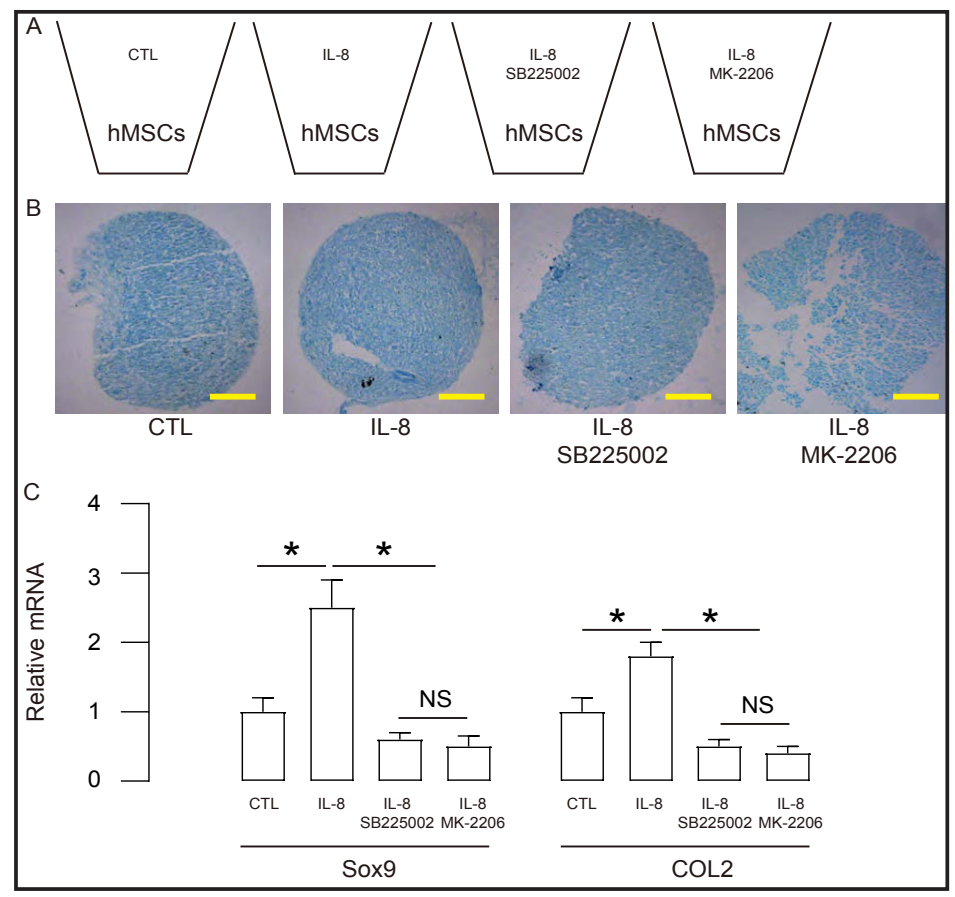

Fig. 2. IL-8 induces chondrogenic differentiation of hBMSCs via CXCR2-mediated PI3k/Akt signaling pathway in vitro. (A) hBMSCs were treated with either control media (CTL), or media with IL-8 (IL8), or media with IL-8 and SB225002 (IL-8+ SB225002), or media with IL-8 and MK-2206 (IL-8+ MK-2206), respectively, and then in vitro chondrogenic differentiation of hBMSCs was assessed in 7 days. (B) Alcian blue staining. (C) RT-qPCR for Sox9 and COL2 in the differentiated hBMSCs. ${ }^{*} \mathrm{p}<0.05$. NS: non-significant. $\mathrm{N}=5$. Scale bars are 50uM.

Fig. 3. Isolation and characterization of mBMSCs. (A) Isolated mouse BMSCs (mBMSCs) in culture. (B) Flow cytometry for Sca-1, CD90, CD105, CD34, CD45 and HLADR in mBMSCs. Red: antibody. Blue: Isotype. (C) chondrocyte differentiation by Alcian blue staining (left), adipocyte differentiation by Oil red 0 staining (middle) and osteocyte differentiation by Von kossa staining (right). $\mathrm{N}=5$. Scale bar is $20 \mathrm{uM}$ in $\mathrm{A}$ and $50 \mathrm{uM}$ in $\mathrm{C}$.
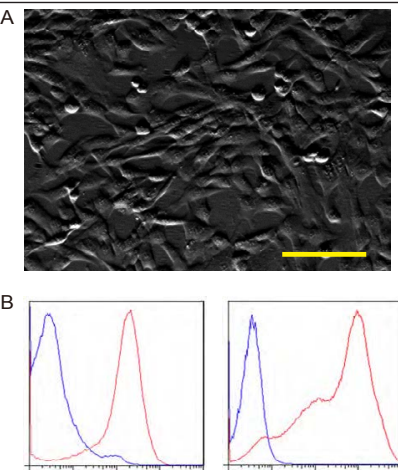

Sca-1

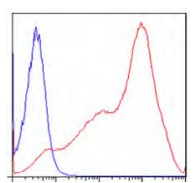

CD90

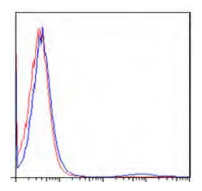

CD34

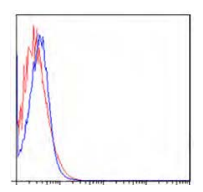

CD45

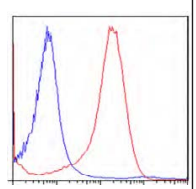
CD105
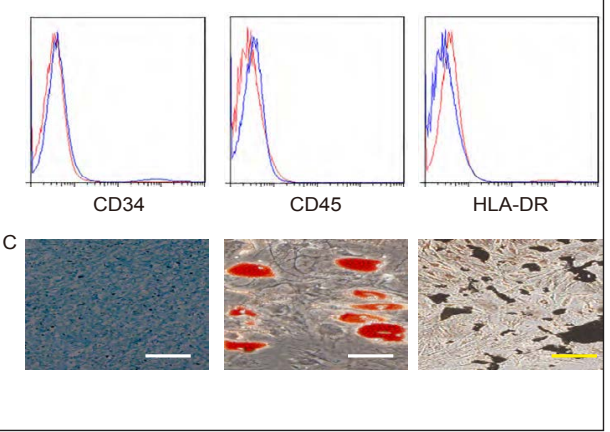


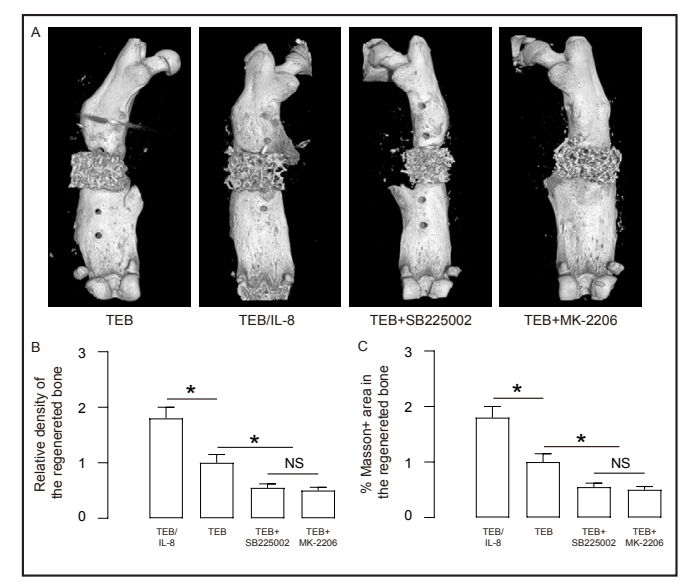

Fig. 4. IL-8 enhances osteogenesis in the TEBimplanted bone defect in mice. (A) Mouse tissue engineered bone (TEB) with/without overexpression of mouse IL-8 (mBMSCs; mBMSCs/IL-8) was prepared (TEB; TEB/IL8). A bone resection was generated at the right femur of the eGFP-transgenic mice, after which TEB, or TEB/IL-8, or TEB with SB225002, or TEB with MK-2206 was implanted into the bone defect. Morphological histological analysis of the samples was taken 14 days after operation by micro-CT. (B) The density of the regenerative bone. (C) Masson staining. ${ }^{*} \mathrm{p}<0.05$. NS: nonsignificant. $\mathrm{N}=10$.

the effects of IL-8 (Fig. 5A-B). These data suggest that IL-8 enhances chondrogenesis via CXCR2-mediated PI3k/Akt signaling pathway in vivo.

\section{Discussion}

Bone regenerative capacity is limited, and is unable to repair large bone defects through its own regeneration. Tissue engineered bone graft is one of the ideal ways to repair large bone defects. At present, the technology of tissue engineering bone is yet perfect, and the mechanism of repairing the bone defect using tissue engineering bone remains poorly understood. A previous study found that seed cells contributed less to the final repair of bone tissue, compared to host-derived cells [12]. Here, we showed that BMSCs on tissue engineering bone release a large amount of IL-8, while some studies have shown that IL-8 is a chemotaxis to MSCs in vitro [33-36]. In this study, transwell experiments were done to confirm that IL-8 has a chemotactic effect on BMSCs. Chemotaxis of IL-8 disappeared after SB225002 treatment on BMSCs but remained after aCXCR1 treatment, suggesting that IL- 8 binds to CXCR2 but not CXCR1 to induce chemotaxis. LY294002 pretreatment of BMSCs inhibited PI3K activity, resulting in disappearance of IL-8 chemotaxis, suggesting that chemotaxis may require activation of PI3K signaling pathway. Moreover, Akt-specific antagonist MK-2206 abolished the effects of IL-8, suggesting that IL-8 enhances bone regeneration via CXCR2mediated PI3k/Akt signaling pathway. Interestingly, the in vivo experimental results verified our findings from in vitro experiments.

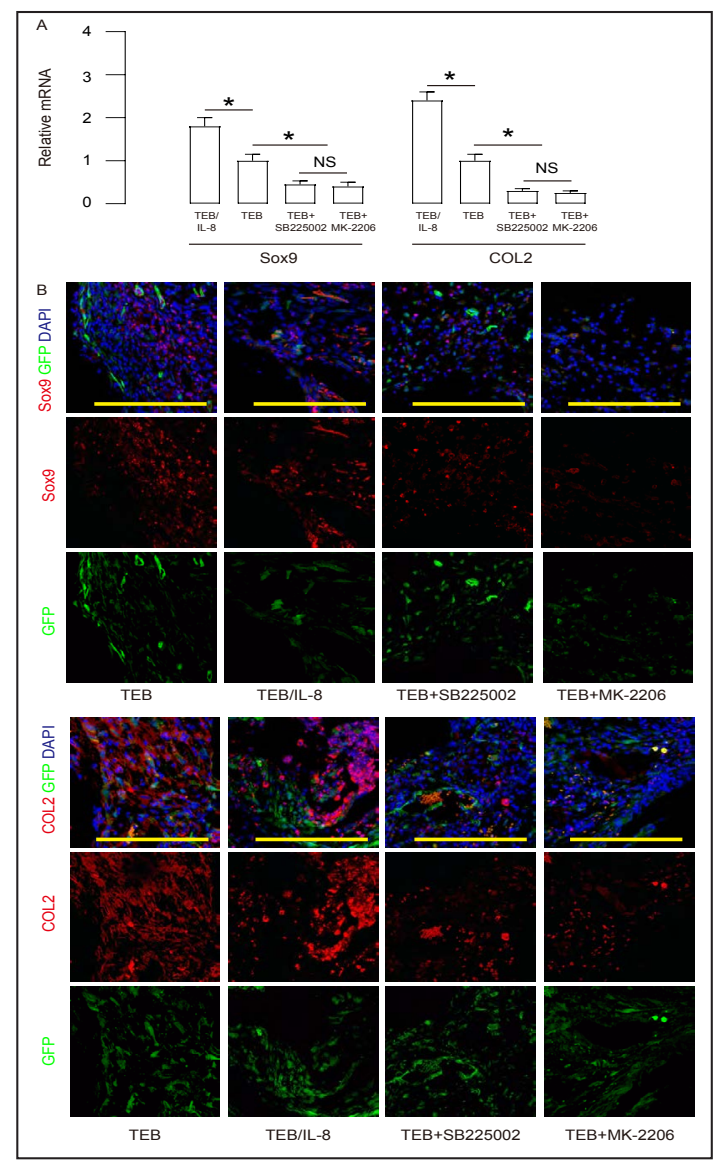

Fig. 5. IL-8 enhances chondrogenesis-associated proteins in the injured mouse bone. (A-B) Chondrogenesis in the regenerated bone was assessed by examination of two chondrogenic

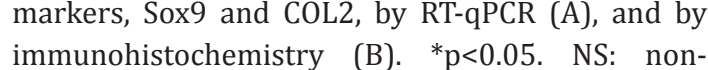
significant. $\mathrm{N}=10$. Scale bars are $50 \mathrm{uM}$.

\section{KARGER}


Endochondral ossification is the mechanism by which most fractures heal. Tissue engineered bone may also repair bone defects through endochondral ossification. Tissue engineered scaffold around the visible cartilage causes collagen accumulation, chondrocytes hypertrophy different from normal chondrocytes. Repairing of bone defects by tissue engineered bone may be initiated by seed cells that secret a variety of cytokines and inflammatory cytokines in the local inflammatory environment. The secreted cytokines may recruit the host cells to the bone defect area, in which the interaction between the seed cells and host cells differentiate into chondrocytes to secrete a large amount of collagen and cartilage matrix, to form cartilage mineralized osteoblast, and induce chondrocyte to osteoblast transformation [37].

Here, we found that tissue engineering bone could repair large segmental defects in animal models and the experimental results showed that IL-8 promote osteogenesis, in a CXCR2/PI3k/Akt-dependent manner.

Bone regeneration primarily occurs through endochondral bone formation, in which mesenchymal condensations determine the bone domain by forming growth cartilage to allow chondrocytes generate and proliferate. The transcription factor Sox 9 is specifically expressed in osteochondroprogenitors in the mesenchymal condensations. Sox9 transcriptionally activates major cartilaginous matrix proteins such as aggrecan and COL2. Thus, Sox9 and COL2 are two markers for the chondrogenic differentiation [38]. Both in vitro and in vivo experiments that analyzed Sox9 and COL2 confirmed the results that IL-8 enhances bone regeneration via CXCR2-mediated PI3k/Akt signaling pathway.

To summarize, here using both in vitro and in vivo tools, we were able to demonstrate a critical role of IL-8 in the bene regeneration through tissue engineering bone technology. Moreover, we found that the involved molecular signaling tranduction was CXCR2-mediated PI3k/Akt signaling pathway.

\section{Acknowledgements}

This study was supported by Natural Science Foundation of China (NO: 81471802) and the Foundation of Southwest Hospital (SWH2015QN05).

\section{Disclosure Statement}

No conflict of interests exists.

\section{References}

1 Miyamoto S, Fujiki M, Setsu N, Kawai A: Simultaneous reconstruction of the bone and vessels for complex femoral defect. World J Surg Oncol 2016;14:291.

$>2$ Nakamura T, Matsumine A, Asanuma K, Matsubara T, Sudo A: Treatment of bone defect with calcium phosphate cement subsequent to tumor curettage in pediatric patients. Oncol Lett 2016;11:247-252.

-3 Hernigou P, Trousselier M, Roubineau F, Bouthors C, Chevallier N, Rouard H, Flouzat-Lachaniette CH: Local transplantation of bone marrow concentrated granulocytes precursors can cure without antibiotics infected nonunion of polytraumatic patients in absence of bone defect. Int Orthop 2016;40:2331-2338.

4 Lin D, Luo D, Lian K, Zhai W, Ding Z: Reconstruction of Traumatic Bone Defect With In Situ Implantation of Dropped Traumatic Segmental Bone Fragments. Orthopedics 2016;39:e14-18.

5 Shah SR, Smith BT, Tatara AM, Molina ER, Lee EJ, Piepergerdes TC, Uhrig BA, Guldberg R, Bennett GN, Wenke JC, Mikos AG: Effects of local antibiotic delivery from porous space maintainers on infection clearance and induction of an osteogenic membrane in an infected bone defect. Tissue Eng Part A 2016

-6 Oryan A, Alidadi S, Moshiri A, Maffulli N: Bone regenerative medicine: classic options, novel strategies, and future directions. J Orthop Surg Res 2014;9:18. 


\section{Cellular Physiology Cell Physiol Biochem 2018;48:361-370 \begin{tabular}{l|l} 
DOI: 10.1159/000491742 & O 2018 The Author(s). Published by S. Karger AG, Basel \\
www.karger.com/cpb
\end{tabular}}

Yang et al.: a Role of IL-8 in BMSCs-Mediated Bone Regeneration

7 Soucacos PN, Kokkalis ZT, Piagkou M, Johnson EO: Vascularized bone grafts for the management of skeletal defects in orthopaedic trauma and reconstructive surgery. Injury 2013;44:S70-75.

8 Guerado E, Fuerstenberg CH: What bone graft substitutes should we use in post-traumatic spinal fusion? Injury 2011;42:S64-71.

-9 Pilipchuk SP, Plonka AB, Monje A, Taut AD, Lanis A, Kang B, Giannobile WV: Tissue engineering for bone regeneration and osseointegration in the oral cavity. Dent Mater 2015;31:317-338.

10 Park BW, Kang EJ, Byun JH, Son MG, Kim HJ, Hah YS, Kim TH, Mohana Kumar B, Ock SA, Rho GJ: In vitro and in vivo osteogenesis of human mesenchymal stem cells derived from skin, bone marrow and dental follicle tissues. Differentiation 2012;83:249-259.

11 Block JE: The role and effectiveness of bone marrow in osseous regeneration. Med Hypotheses 2005;65:740-747.

12 Hou T, Li Q, Luo F, Xu J, Xie Z, Wu X, Zhu C: Controlled dynamization to enhance reconstruction capacity of tissue-engineered bone in healing critically sized bone defects: an in vivo study in goats. Tissue Eng Part A 2010;16:201-212.

13 Tortelli F, Tasso R, Loiacono F, Cancedda R: The development of tissue-engineered bone of different origin through endochondral and intramembranous ossification following the implantation of mesenchymal stem cells and osteoblasts in a murine model. Biomaterials 2010;31:242-249.

14 Tasso R, Augello A, Boccardo S, Salvi S, Carida M, Postiglione F, Fais F, Truini M, Cancedda R, Pennesi G: Recruitment of a host's osteoprogenitor cells using exogenous mesenchymal stem cells seeded on porous ceramic. Tissue Eng Part A 2009;15:2203-2212.

15 Zhou Y, Fan W, Prasadam I, Crawford R, Xiao Y: Implantation of osteogenic differentiated donor mesenchymal stem cells causes recruitment of host cells. J Tissue Eng Regen Med 2015;9:118-126.

-16 Roh JD, Sawh-Martinez R, Brennan MP, Jay SM, Devine L, Rao DA, Yi T, Mirensky TL, Nalbandian A, Udelsman B, Hibino N, Shinoka T, Saltzman WM, Snyder E, Kyriakides TR, Pober JS, Breuer CK: Tissue-engineered vascular grafts transform into mature blood vessels via an inflammation-mediated process of vascular remodeling. Proc Natl Acad Sci U S A 2010;107:4669-4674.

17 Hibino N, Villalona G, Pietris N, Duncan DR, Schoffner A, Roh JD, Yi T, Dobrucki LW, Mejias D, Sawh-Martinez R, Harrington JK, Sinusas A, Krause DS, Kyriakides T, Saltzman WM, Pober JS, Shin'oka T, Breuer CK: Tissueengineered vascular grafts form neovessels that arise from regeneration of the adjacent blood vessel. FASEB J 2011;25:2731-2739.

18 Tasso R, Fais F, Reverberi D, Tortelli F, Cancedda R: The recruitment of two consecutive and different waves of host stem/progenitor cells during the development of tissue-engineered bone in a murine model. Biomaterials 2010;31:2121-2129.

19 Hoffmann E, Dittrich-Breiholz 0, Holtmann H, Kracht M: Multiple control of interleukin-8 gene expression. J Leukoc Biol 2002;72:847-855.

20 Yoshimura T: Discovery of IL-8/CXCL8 (The Story from Frederick). Front Immunol 2015;6:278.

21 Britschgi A, Radimerski T, Bentires-Alj M: Targeting PI3K, HER2 and the IL-8/JAK2 axis in metastatic breast cancer: Which combination makes the whole greater than the sum of its parts? Drug Resist Updat 2013;16:68-72.

-22 Ringe J, Strassburg S, Neumann K, Endres M, Notter M, Burmester GR, Kaps C, Sittinger M: Towards in situ tissue repair: human mesenchymal stem cells express chemokine receptors CXCR1, CXCR2 and CCR2, and migrate upon stimulation with CXCL8 but not CCL2. J Cell Biochem 2007;101:135-146.

23 Hu H, Chen M, Dai G, Du G, Wang X, He J, Zhao Y, Han D, Cao Y, Zheng Y, Ding D: An Inhibitory Role of Osthole in Rat MSCs Osteogenic Differentiation and Proliferation via Wnt/beta-Catenin and Erk1/2-MAPK Pathways. Cell Physiol Biochem 2016;38:2375-2388.

24 Choi JW, Shin S, Lee CY, Lee J, Seo HH, Lim S, Lee S, Kim IK, Lee HB, Kim SW, Hwang KC: Rapid Induction of Osteogenic Markers in Mesenchymal Stem Cells by Adipose-Derived Stromal Vascular Fraction Cells. Cell Physiol Biochem 2017;44:53-65.

25 Dong K, Hao P, Xu S, Liu S, Zhou W, Yue X, Rausch-Fan X, Liu Z: Alpha-Lipoic Acid Alleviates High-Glucose Suppressed Osteogenic Differentiation of MC3T3-E1 Cells via Antioxidant Effect and PI3K/Akt Signaling Pathway. Cell Physiol Biochem 2017;42:1897-1906.

-26 Huang G, Kang Y, Huang Z, Zhang Z, Meng F, Chen W, Fu M, Liao W, Zhang Z: Identification and Characterization of Long Non-Coding RNAs in Osteogenic Differentiation of Human Adipose-Derived Stem Cells. Cell Physiol Biochem 2017;42:1037-1050. 


\section{Cellular Physiology Cell Physiol Biochem 2018;48:361-370 \begin{tabular}{l|l} 
DOI: 10.1159/000491742 & O 2018 The Author(s). Published by S. Karger AG, Basel \\
www.karger.com/cpb
\end{tabular} \\ Yang et al.: a Role of IL-8 in BMSCs-Mediated Bone Regeneration}

27 Wu J, Wang C, Miao X, Wu Y, Yuan J, Ding M, Li J, Shi Z: Age-Related Insulin-Like Growth Factor Binding Protein-4 Overexpression Inhibits Osteogenic Differentiation of Rat Mesenchymal Stem Cells. Cell Physiol Biochem 2017;42:640-650.

28 Wang H, Xie Z, Hou T, Li Z, Huang K, Gong J, Zhou W, Tang K, Xu J, Dong S: MiR-125b Regulates the Osteogenic Differentiation of Human Mesenchymal Stem Cells by Targeting BMPR1b. Cell Physiol Biochem 2017;41:530-542.

29 Wang J, Liao J, Zhang F, Song D, Lu M, Liu J, Wei Q Tang S, Liu H, Fan J, Zou Y, Guo D, Huang J, Liu F, Ma C, Hu X, Li L, Qu X, Chen L, Weng Y, Lee MJ, He TC, Reid RR, Zhang J: NEL-Like Molecule-1 (Nell1) Is Regulated by Bone Morphogenetic Protein 9 (BMP9) and Potentiates BMP9-Induced Osteogenic Differentiation at the Expense of Adipogenesis in Mesenchymal Stem Cells. Cell Physiol Biochem 2017;41:484-500.

-30 Bahney CS, Hu DP, Taylor AJ, Ferro F, Britz HM, Hallgrimsson B, Johnstone B, Miclau T, Marcucio RS: Stem cell-derived endochondral cartilage stimulates bone healing by tissue transformation. J Bone Miner Res 2014;29:1269-1282.

31 Park MS, Kim YH, Jung Y, Kim SH, Park JC, Yoon DS, Kim SH, Lee JW: In Situ Recruitment of Human Bone Marrow-Derived Mesenchymal Stem Cells Using Chemokines for Articular Cartilage Regeneration. Cell Transplant 2015;24:1067-1083.

32 Szade K, Zuba-Surma E, Rutkowski AJ, Jozkowicz A, Dulak J: CD45-CD14 +CD34 + murine bone marrow lowadherent mesenchymal primitive cells preserve multilineage differentiation potential in long-term in vitro culture. Mol Cells 2011;31:497-507.

-33 Liu CJ, Kuo FC, Wang CL, Kuo CH, Wang SS, Chen CY, Huang YB, Cheng KH, Yokoyama KK, Chen CL, Lu CY, Wu DC: Suppression of IL-8-Src signalling axis by 17beta-estradiol inhibits human mesenchymal stem cellsmediated gastric cancer invasion. J Cell Mol Med 2016;20:962-972.

-34 Yoon DS, Lee KM, Kim SH, Kim SH, Jung Y, Kim SH, Park KH, Choi Y, Ryu HA, Choi WJ, Lee JW: Synergistic Action of IL-8 and Bone Marrow Concentrate on Cartilage Regeneration Through Upregulation of Chondrogenic Transcription Factors. Tissue Eng Part A 2016;22:363-374.

-35 Wang J, Wang Y, Wang S, Cai J, Shi J, Sui X, Cao Y, Huang W, Chen X, Cai Z, Li H, Bardeesi AS, Zhang B, Liu M, Song W, Wang M, Xiang AP: Bone marrow-derived mesenchymal stem cell-secreted IL-8 promotes the angiogenesis and growth of colorectal cancer. Oncotarget 2015;6:42825-42837.

-36 Zhou X, Gu J, Gu Y, He M, Bi Y, Chen J, Li T: Human Umbilical Cord-Derived Mesenchymal Stem Cells Improve Learning and Memory Function in Hypoxic-Ischemic Brain-Damaged Rats via an IL-8-Mediated Secretion Mechanism Rather than Differentiation Pattern Induction. Cell Physiol Biochem 2015;35:2383-2401.

37 Harada N, Watanabe Y, Sato K, Abe S, Yamanaka K, Sakai Y, Kaneko T, Matsushita T: Bone regeneration in a massive rat femur defect through endochondral ossification achieved with chondrogenically differentiated MSCs in a degradable scaffold. Biomaterials 2014;35:7800-7810.

-38 Ono N, Ono W, Nagasawa T, Kronenberg HM: A subset of chondrogenic cells provides early mesenchymal progenitors in growing bones. Nat Cell Biol 2014;16:1157-1167. 